\title{
Zur Behandlung der Geburt in Schädellage bei nach hinten gerichtetem Hinterhaupte.
}

Nach einem in Berlin anf dem X. internationalen medicinischen Congresse gehaltenen Vortrage.

Von

Dr. Johannes Meyer zu Dorpat.

Der Verlauf der Geburt in Schädellage bei nach hinten gerichteter kleiner Fontanelle pflegt namentlich bei Erstgebärenden ein sehr verschleppter zu sein. Es liegt fernerhin die Möglichkeit vor, dass der regelmässige Mechanismus der Geburt nicht eingehalten wird und der Austritt des Kopfes in der als pathologisch zu bezeichnenden Vorderscheitellage erfolgt.

Die mit der längeren Dauer der Geburt verknüpften Gefahren sind bei Geburten, welche unter ärztlicher Aufsicht verlaufen, durch Beobachtung der aseptischen Cautelen zwar nur noch auf die Schwächung der Mutter und des Kindes durch die ungebührlich lange Geburtsarbeit zu beziehen, aber es wäre vom humanen Standpunkte aus von hohem Werthe, wenn es Mittel gäbe, den Geburtsverlauf in unschädlicher Weise zu beschleunigen.

Die bisher üblichen Verfahren der Kunsthülfe zu diesem Zwecke müssen als durchaus unzulänglich bezeichnet werden.

So gab Lan ge 1844 (Prager Vierteljahrsschrift II, S. 53) den Rath, mittels des Forceps den Kopf in demselben schrägen Beckendurchmesser, in welchem die Pfeilnaht verläuft, so zu fassen, dass der eine Löffel an der mehr nach vorn gerichteten Seite der Stirn, der andere an der entgegengesetzten Seite des Hinterhauptes liegt. Wird nun die Zange angezogen und gleichzeitig in den queren Durchmesser gedreht, so kommt die Pfeilnaht in den queren Durchmesser, das Hinterhaupt mehr nach vorn und tritt schliesslich in regelmässiger Weise aus.

Scanzoni wollte (1867, Lehrbuch der Geburtshülfe, IV. Auflage, Bd. III, S. 170) die Zange in dem schrägen Durchmesser, in welchem die Pfeilnaht nicht verläuft, anlegen und den Kopf zunächst so drehen, dass die Zange in den geraden Durchmesser 
gelangt. Ist dann die Pfeilnaht in den queren Durchmesser gebracht, so wird die Zange zum zweiten Male in dem anderen schrägen Durchmesser, in welchem ursprünglich die Pfeilnaht verlief, angelegt und der Kopf unter Rotation und Traction mit nach vorn gerichteter kleiner Fontanelle entwickelt.

Diese Verfahren fanden wenig Anklang, ja Schröder warnt ernstlich vor der Zangenoperation, so lango die kleine Fontanelle nicht der vorderen Hälfte des knöchernen Beckenringes anliegt. Es soll der natïrliche Eintritt der Drehung abgewartet und bei dringender Lebensgefahr der Kopf ohne vorausgegangene Drehung in der Vorderscheitellage entwickelt werden.

In Fehling's Bearbeitung der geburtshülflichen Operationen (Müller's Handbuch der Geburtshülfe, Abschnitt X, S. 141) wird dieselbe Auffassung vertreten, nur bei strengster Anzeige zur Zangenanlegung den Kopf in Vorderscheitellage zu entwickeln. Werth (dasselbe Handbuch, Abschnitt IV, die Physiologie der Geburt betreffend, S. $400 \mathrm{ff}$.) behandelt eingehend die Bedeutung dieser Lagen, ohne auf die Behandlung näher einzugehen.

Es ist daher wohl gerechtfertigt, wenn ich drei von mir beobachtete einschlägige Fälle in aller Kürze mittheile, in welchen es mir gelang, die innere Drehung des Kopfes durch combinirte Handgriffe von aussen her und von der Scheide aus zu erreichen.

Den ersten Fall beobachtete ich 1882 als Assistent der Universitäts-Entbindungsanstalt in der Poliklinik zu Dorpat. Die Veröffentlichung gestattete mir gütigst der gegenwärtige Director Herr Prof. O. Küstner. Die beiden anderen Fälle kamen 1889 in meiner Privatpraxis vor.

F all I. 17. October 1882. E. A., 22 Jahre alt, Estin, Erstgebärende. I. Schädellage, II. Unterart (Rücken links hinten), Becken regelmässig. Beginn der Wehen $11 \mathrm{Uhr}$ Abends am 15. October. Am Abende des 16. October fand ich den Muttermund auf 5-6 cm eröffnet, nachgiebig; Kopf in den Beckeneingang eingetreten, grosse Fontanelle nach vorn rechts, kleine Fontanelle nach hinten, etwas links. Kopf leicht beweglich, Blase steht. Wehen anhaltend, aber sehr schwach. Um 2 Uhr Morgens des 17. October Muttermund noch weiter eröffnet, Lage und Beweglichkeit des Kopfes unverändert. Durch Druck auf den Gebärmuttergrund gelingt es, eine Drehung des Kopfes in dem Sinne zu bewirken, dass die kleine Fontanelle allmälig nach links, schliesslich sogar mehr nach vorn trat, - die Pfeilnaht somit aus dem zweiten schrägen Durchmesser in den ersten gebracht wurde. "Bei Nachlass des Druckes schnellte der Kopf wieder in die ursprïngliche Stellung zurïck. Ich entschloss mich daher, nachdem durch ausgiebigen Druck auf den Steiss des Kindes in der Richtung. der Längenachse der Gebärmutter die kleine Fontanelle wieder nach 
vorn gebracht und in dieser Stellung durch anhaltenden Druck festgestellt worden, die Zange anzulegen. Die Ausziehung war leicht, der Kopf ward in regelmässiger Hinterhauptslage entwickelt, das Kind, ein reifes Mädchen, kam lebend zur Welt, das Wochenbett verlief fieberlos. Aus meinen Niederschriften geht es leider nicht hervor, wann die Blase gesprungen war.

Fall II. 11. October 1889. M. von M., 27 Jahre alt, Deutsche, Erstgebärende. Im Jahre vorher Abort in der zehnten Schwangerschaftswoche. II. Schädellage, II. Unterart (Rücken rechts hinten), Becken regelmässig. Am Abende des 10. October Beginn der Wehen, welche sehr schwach wirkten. Am Abende des 10. October war der Muttermund erst auf $3 \mathrm{~cm}$ eröffnet, die Blase spannte sich bei den Wehen fast gar nicht. Obschon die Pfeilnaht im ersten schrägen Durchmesser stand, sprengte ich die Blase. Es entleerte sich eine geringe Menge Fruchtwasser; die Wehen wurden stärker. Der Muttermund erweiterte sich, die Pfeilnaht stellte sich quer. Um 6 Uhr Morgens am 11. October hatte sich letztere wieder in den ersten schrägen Durchmesser zurückgedreht. Bei der Untersuchung bemerkte ich, dass der Kopf bei geeignetem Drucke aufs Hinterhaupt, verbunden mit sanftem Drucke auf das ïber dem horizontalen Schambeinaste tastbare Kinn in der Richtung nach hinten, sich in der Weise umdrehen liess, dass die kleine Fontanelle sich nach vorn wandte. Der Kopf drehte sich zurück, sobald die mechanische Einwirknng anf denselben unterbrochen ward. Unter gütiger Mitwirkung des Herrn Docenten Dr. L. Kessler gelang es, den Kopf durch Druck auf das Kinn in der Richtung nach hinten, auf den Steiss in der Richtung nach unten und durch Zug und Druck auf das Hinterhaupt von der Scheide aus so zu drehen, dass die Pfeilnaht in den zweiten schrägen Durchmesser gelangte. Ich legte unter beständigem Fithalten des Kopfes in der gewonnenen Lage die Zange an, welche tadellos regelrecht dem Kopfe anlag. Letzterer stand tief im Beckeneingange. Bei der ersten Traction stellte sich die Pfeilnaht vollständig in den geraden Durchmesser, die Ausziehung beanspruchte sechs Tractionen. Der Damm unverletzt, das Kind, ein kräftiges Mädchen, kam lebend zur Welt, das Wochenbett verlief regelmässig.

Fall III. 19. October 1889. E. G., 29 Jahre alt, Deutsche, Erstgebärende. II. Schädellage, II. Unterart (Rücken rechts hinten), Becken regelmässig. Beginn schwacher Wehen am Morgen des 18. October. Um 6 Uhr Nachmittags setzten heftigere Weben ein. Um 1 Uhr Morgens am 19. October war der Muttermund fast verstrichen, die Pfeilnaht im ersten schrägen Durchmesser. Grosse Fontanelle bequem zu erreichen, die kleine Fontanelle hochstehend, nicht abzutasten. Der Kopf tief im Beckeneingange, Wehen kräftig. Durch Druck auf das Hinterhaupt von hinten rechts nach vorn rechts, Druck auf das Kinn and die Beine der Frucht im Sinne einer Drehung nach links hinten gelang es, die Pfeilnaht in den queren Durchmesser zu bringen, nach 15 Minuten anhaltenden Bemühungen sogar in den zweiten sehrägen Durchmesser zu drängen. Nun ward die Blase gesprengt und der Kopf mittels der Hand in der neuen Stellung fest-. 
gehalten. Unter starken Wehen trat die kleine Fontanelle tiefer herab, die grosse Fontanelle war micht mehr zu erreichen, der Kopf stand fest, ohne Neigung, wieder zurückzuweichen. Die Geburt musste schliesslich wegen Wehenschwäche in der Austreibungsperiode durch Kunsthülfe beendet werden. Damm unverletzt, leichte Ausziehung. Lebendes Mädehen von $4,4 \mathrm{~kg}$ Gewicht; Umfang des Schädels über Glabella-Occiput gemessen $38,0 \mathrm{~cm}$. Wochenbett regelmässig.

Die Erfahrung, welche ich in diesen drei Fällen gemacht, veranlasst mich, das von mir angewandte Verfahren warm zu empfehlen. Dasselbe ist nur bei regelmässigen Grössenverhältnissen des Beckens, sowie des kindlichen Schädels anzuwenden. Bei Regelwidrigkeiten des Beckens hat die Einstellung des Schädels nur secundäre Bedeutung; bei der Behandlung kommt massgebend nur die Beckenabweichung, weniger die Lage der kleinen Fontanelle in Betracht. Um das Verfahren erfolgreich anwenden zu können, muss der Muttermund bequem für zwei Finger durchgängig sein. Es ist rathsam, mit den Handgriffen erst dann zu beginnen, sobald der Kopf mit seinem grössten Umfange in den Beckeneingang eingetreten ist. Die Einleitung der Narkose, die oft nur eine oberflächliche zu sein braucht, wird ausnahmelos den Erfolg des Eingriffes in hohem Grade begünstigen und dürfte wenig Einwände finden. Die strenge Beobachtung aseptischer Vorsichtsmassregeln, nöthigenfalls eine vorausgeschickte Desinfection des Geburtscanals sind unerlässliche Massregeln. Bei den Handgriffen innerhalb der Scheide wird, soweit es möglich ist, die Blase geschont. Bei nach rechts liegender kleiner Fontanelle wird die linke, im anderen Falle die rechte Hand zur Ausfïhrung der Handgriffe in der Scheide gewählt. Die halbe Hand, bei enger Scheide zwei Finger, werden in der Ausbuchtung des kleinen Beckens, welche der Synchondrosis sacro-iliaca entspricht, möglichst hoch am Hinterhaupte hinaufgeführt, wobei es in einzelnen Fällen zweckdienlich sein dürfte, den Kopf ein wenig hinaufzudrängen und dadurch zu lüften. Durch die an das Hinterhaupt angelegten Finger soll ein ruhiger gleichmässiger Druck in der Richtung nach vorn und unten ausgeübt werden. Der Neigung der grossen Fontanelle, infolge des Zuges nach unten tiefer zu treten, arbeitet man zugleich durch Druck auf das Hinterhaupt in der Richtung des Querdurchmessers des Beckens entgegen. Die äussere Hand des Operateurs schiebt das über dem Schambeinaste fühlbare Kinn nach hinten, wobei ein Druck nach unten sorgfältig zu vermeiden ist. Ein Gehülfe, oder in Ermangelung eines solchen die Hebamme, trachtet durch Druck auf den Steiss 
der Frucht in Richtung der Gebärmutterachse, sowie durch Zurückdrängen der Beine nach hinten die angestrebte Drehbewegung auch dem Körper des Kindes mitzutheilen. Welcher der erwähnten Handgriffe besser wirkt und somit kräftiger auszuiuben ist kann durch die in der Scheide liegende Hand stets beobachtet und im einzelnen Falle nach Bedürfniss geregelt werden.

Erst nach anhaltenden erfolglosen Bemühungen darf das Verfahren als unwirksam verlassen werden. Ist jedoch die Drehung gelungen, so lege man sogleich die Zange an den künstlich festgestellten Kopf an; mangelt die Anzeige zur Beendigung der Geburt, so ist unter steter Festhaltung des Kopfes die Wehenthätigkeit anzuregen, welche bald die gewonnene günstige Einstellung sichern kann. Namentlich bei Erstgebärenden kann das Verfahren, welches bei einiger Behutsamkeit als völlig ungefährlich bezeichnet werden muss, die Dauer der Geburtsarbeit wesentlich verkürzen.

Ich erachte es für meine Pflicht, zum Schlusse Folgendes zu erwähnen. Erst nachdem ich auf dem X. internationalen Congresse zu Berlin meine Beobachtungen mitgetheilt hatte, fand ich unlängst eine Notiz, welche mich überzeugte, dass ich für das von mir geschilderte Verfahren blos die Originalität für mich in Anspruch nehmen darf. Die Priorität der Veröffentlichung gebührt Herrn Emil Blanc, dessen Aufsatz: ,de la correction manuelle des occipito-postérieures persistantes“" bereits 1887 (Lyon méd. Nr. 3, 4 und 6, S. 73-83, 110-118, 184-193) veröffentlicht worden ist. Es ist mir leider nicht gelungen, in den Besitz der Originalabhandlung zu kommen, und muss ich daher auf die kurzen Inhaltsangaben verweisen, welche sich im I. Jahrgange der Jahresberichte über die Fortschritte der Geburtshülfe und Gynäkologie, S. 57 und 137 finden. Meine erste Beobachtung fällt jedoch bereits in das Jahr 1882, so dass damit bewiesen ist, dass ich unabhängig von Herrn E. Blanc auf dieses Verfahren aufmerksam geworden bin.

Wenn ich trotz der von E. Blanc veröffentlichten Arbeit doch noch meine Fälle zur Veröffentlichung bringe, so geschieht es erstens, weil das Verfahren in Deutschland, wie es mir scheint, noch wenig bekannt geworden, und zweitens, weil ich durch die daran geknüpfte Schlussbemerkung dem Vorwurfe vorbeugen will, dass ich durch meine Mittheilung auf dem X. internationalen Congresse in Berlin absichtlich die Priorität für mich hätte in Anspruch nehmen wollen. 